\title{
Estudio de los partidos de izquierda en Santa Cruz (1983-2015)
}

Study of the left-wing parties in Santa Cruz (1983-2015)

\author{
Daniela Mayer, danielacmayer@outlook.com \\ Juan Ramón Vilaboa, vilaboa_5@hotmail.com \\ Mirna Susana Hudson, mirnahudson64@gmail.com \\ Departamento de Humanidades y Ciencias Sociales
}

Universidad Nacional de la Patagonia Austral, Unidad Académica de Río Gallegos

Av. Piloto Rivero s/n. Río Gallegos, Santa Cruz, Argentina

Recibido: 22/03/2017. Aceptado: 30/07/2018

\section{RESUMEN}

El objetivo del trabajo es analizar las formaciones partidarias de izquierda en la provincia de Santa Cruz como espacios de discusión en el entramado político, teniendo en cuenta su estructuración como partido, la forma de organización y su accionar partidario en los diferentes escenarios.

El trabajo se basa en una búsqueda en diferentes diarios provinciales, recopilación de resultados electorales y realización de entrevistas a los protagonistas. Con ello se pretende llegar a conocer la dinámica de organización interna y el entramado de relaciones que tejieron estos partidos, para luego mantenerse como minoría activas en el escenario político santacruceño.

Palabras clave: Elecciones, Santa Cruz, Organización, Participación.

\begin{abstract}
The left-wing parties, that were appreciated as political minorities in terms of elections, had established in the centre of the politic discussion because of their interventions in the society. The objective of this research work is to analyze the creation of left-wing parties in the province of Santa Cruz as discussion spaces in the political web, having into account their organization as party, their ways of planning and act on different social conflicts. By means of a search in different local newspapers, compilations of election results and carry out interviews, we can conclude to a dynamic of inner organization in the developing of these parties, understanding why they still as an alternative for the electorate.
\end{abstract}

Key words: elections, Santa Cruz, organization, involvement.

\section{INTRODUCCIÓN}

Este informe es el resultado de una beca desarrollada en el marco del grupo de investigación "Las formas de la política en una perspectiva de larga duración. Proyectos políticos, configuraciones y prácticas entre 1912-2013"de la UNPA. La misma estuvo enfocada a analizar los partidos de izquierda en Santa Cruz desde 1983 a la fecha. 
El panorama político del regreso de la vida partidaria se amplió con la participación de entidades de origen ideológico distinto, generando así una línea de trabajos en relación con aquellos partidos tradicionales a nivel nacional, dado los grandes cambios y crisis internas por las que atravesaron. Pero en el caso de la provincia de Santa Cruz, pocos son los antecedentes de estudios sobre el papel de los partidos de izquierda luego de 1983, tanto en su organización como en las elecciones provinciales.

La izquierda comienza a transformarse en un tema de estudio en vías de consolidación, representando una novedad para la historia política de Santa Cruz. El objetivo general del presente trabajo se enfoca en analizar las formaciones partidarias de izquierda presentes en la provincia, las acciones políticas que impulsan, las problemáticas que incorporan en su discurso, como así también las estrategias y alternativas empleadas para instalarse en el debate político provincial. El análisis de los resultados electorales, de los diarios provinciales y entrevistas a militantes, serán fuentes utilizadas para abordar el estudio de las propuestas hechas por parte de los partidos y la aceptación de las mismas en la sociedad.

Es entonces que la estructuración de este trabajo comenzará con la composición del marco teórico, autores escogidos para explicar la organización interna de un partido político, siguiendo con la exposición de los resultados por medio de tablas y los temas utilizados por los partidos. Así también, se realizará la discusión de los puntos presentados para su comparación entre la teoría y los resultados expuestos.

Finalmente, en la conclusión, se hará un cierre de las ideas sobre la estructura de un partido de izquierda y su forma de organización frente a las demandas de la sociedad, para resolver y dar propuestas o soluciones, actualizando las condiciones por sus principales medios de difusión.

\section{MARCO TEÓRICO}

Para comenzar con el trabajo, se debían responder a un par de preguntas orientativas ante la novedad de los partidos de izquierda y su presencia a partir de 1983: ¿cuál es la estructura de un partido?, ¿a quién se considera miembro de uno?, ¿qué papel deben cumplir estos partidos?, ¿de qué forma dan respuestas a los problemas sociales? Y finalmente ¿cómo actúan durante las épocas de elecciones? Partiendo de estas incógnitas, las ideas de los siguientes autores fueron utilizadas como base.

Maurice Duverger plantea que el desarrollo de los partidos parece estar relacionado con la democracia y que, cuanto más ven crecer sus funciones, más sienten sus miembros la necesidad de agruparse (Duverger 1952). La vida política que se llega a manifestar en el partido, tendrá una mayor importancia según las actividades y los fines para llegar a cargos políticos.

A continuación, Duverger realiza una diferenciación en el desarrollo de los nuevos partidos (en base al contexto por el cual escribe el autor) por sobre los tradicionales o antiguos: la importancia de nuevos electores. No bastan las iniciativas locales sin el apoyo constante de los grupos internos de un partido y la búsqueda de nuevos miembros, cuestión importante para su permanencia en el tiempo.

A partir de lo expuesto, habría que pensar de qué forma los partidos de izquierda proceden para diferenciarse de los demás partidos, y así adquirir una mayor captación del electorado o futuros miembros.

Continuando con Duverger, la importancia de la composición de un partido es la distinción de los miembros estables, es decir, de aquellos que han firmado una adhesión hacia el partido (Duverger 1952). Para estos partidos, la adhesión de miembros es la manera de continuar con su existencia y presentarse a elecciones, acción importante para poder mantenerse y que el 
mismo autor resalta "¿habrá que distinguir dos clases de partidos: los partidos tradicionales, relativamente poco sensibles a las "variaciones cíclicas", y los partidos nuevos, que fundan en ellas su crecimiento o su caída?" (Duverger 1952, p.114).Diferencia así tres círculos de participación: electores, "simpatizantes" y militantes, roles que aparecerán en épocas de elecciones y se mantendrán luego de ellas.

Por otra parte, para presentar la estructura interna de un partido, Giovanni Sartori diferencia cuatro dimensiones:

1- de organización,

2- de motivaciones,

3- ideológica y,

4- de izquierda-derecha.

La primera tiene una variación independiente, dando como ejemplo de organización la disposición de la prensa. La dimensión de motivación "requiere toda una batería de indicadores, observaciones a lo largo de mucho tiempo y el método de la reputación" (Sartori 1980, p. 104). Por otra parte, la ideológica mantiene relaciona con la motivación, siendo un aspecto de fuerza para la organización interna. Para el autor, esta tercera dimensión difiere de las demás al señalar un factor cultural, enfocado en el ánimo general de la política (Sartori 1980), finalmente la última dimensión refiere a la tendencia por la cual un partido elige una percepción que pueda caracterizar al movimiento.

Para el caso trabajado, como es la estructura de los partidos de izquierda y su diferenciación con los demás partidos tradicionales, Osvaldo Coggiola describe aspectos sobre la dinámica de estos partidos. Por medio de una serie de punteos acerca de cómo el trotskismo se instaló en América Latina y en Argentina, caracteriza que "un rasgo de gran importancia es que algunas de sus expresiones más avanzadas muestran cómo, en los grandes procesos de movilización social, se hace sentir la necesidad de formas más amplias y democráticas de organización para la lucha y de métodos más radicales de acción.” (Coggiola 2006, p. 466). Nos plantea que, si bien, los partidos de izquierda no influencian de manera directa en la forma de organización en los momentos de lucha del movimiento obrero, se necesita de la participación de estos partidos en ciertos procesos de movilización para el apoyo y representación de las necesidades de los trabajadores.

Para el autor, "la historia del marxismo revolucionario, en la medida en que es la expresión conciente de la tendencia inconciente del proletariado a reestructurar la sociedad sobre bases socialistas, no es nunca independiente de la historia del movimiento obrero, aunque su influencia organizada sobre éste sea mínima" (Coggiola 2006, p. 339).

Teniendo en cuenta el marco presentado, se tomó para analizar el entramado político de la provincia de Santa Cruz, el libro de Horacio Lafuente sobre la actividad política en la provincia. Si bien su análisis llega hasta a 1985, lo importante de su libro son los datos electorales que el autor nos presenta, rescatando y conformando los primeros números en las elecciones de 1983 para la izquierda.

\section{Método de abordaje}

Para el trabajo, se analizaron los Diarios de Correo del Sur y la Opinión Austral de los años 1983, 1985, 1995, 2007 y 2015. Fuentes electorales de los años 1983, 1985, 1987, 1989, 1991, 1993, 1995, 1997, 1999, 2003, 2007, 2011 y 2015. Así también, se realizó una entrevista a Miguel del Pla, representante del Partido Obrero en Santa Cruz. 


\section{RESULTADOS}

Para las elecciones de 1983 en Santa Cruz, se presentaron a las categorías de gobernadorvicegobernador y diputados provinciales, el Partido Justicialista y la Unión Cívica Radical, como principales candidatos a ganar las elecciones, resultando triunfador en las mismas el Partido Justicialista. También se postularon el Movimiento de Integración y Desarrollo, el Partido Intransigente, el Frente de Izquierda Popular y el Partido Socialista Popular. Se tomaron así, los siguientes partidos de izquierda para su análisis:

- Partido Obrero

- Movimiento al Socialismo

- Partido Comunista

Los primeros resultados que se observan de la investigación son las variaciones de los porcentajes en las elecciones en las categorías de diputados provinciales (tablas 2, 4, 6 y 8),concentrados en su mayoría en esta primera categoría, a diferencia de la categoría de gobernador y vicegobernador (tablas 1, 3, 5 y 7), presentando una leve variación.

A continuación, en la tabla 1 pueden observarse los primeros resultados electorales durante el proceso a la vuelta de la Democracia en el país. En la provincia de Santa Cruz, para la categoría de Gobernador y Vicegobernador, se contaron un total de 44.556 votos, de los cuales 135 fueron para el Movimiento al Socialismo (a partir de ahora MAS) y 154 para el Partido Obrero (a partir de ahora PO).En una nueva elección en 1987, en un total de 58.873 votantes, el Movimiento al Socialismo obtuvo 297 votos y el Partido Obrero 313 votos. Un detalle a resaltar es que para la categoría presentada en la tabla 1, el Partido Comunista (a partir de ahora PC) decide no presentar candidatos.

Tabla 1

Resultados elecciones '80 de Gobernador y Vicegobernador*

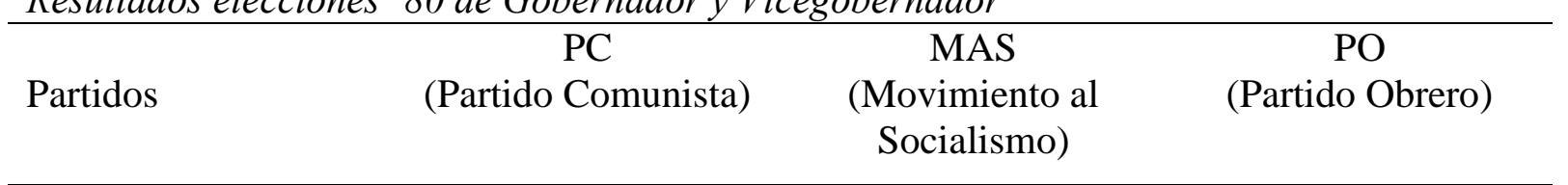

\begin{tabular}{lccc}
\hline Años & & & \\
\hline 1983 & - & 0,3 & 0,4 \\
\hline 1987 & - & 0,5 & 0,5
\end{tabular}

*Nota: Tabla de creación propia. Resultados tomados de Lafuente (2007) y Dirección Nacional Electoral.

Por otra parte, en la tabla 2 para la categoría de Diputados Provinciales, se registraron un total de 44.556 votos, siendo de ellos 259 para el PC, 151 para el MAS y 149 para el PO. Para 1985, de los 51.089 votos registrados, solo 308 fueron para el PO, remarcando solo la participación de este último partido izquierda.

En 1987, hay una nueva presentación del PO y el MAS. Se registra un un total de 59.300 votantes, 336 votos fueron para el primero y 277 para el segundo. Para finales de los ochenta, en las elecciones de 1989, una vez más, solo se presenta el PO, obteniendo 556 votos en un total de 65.486 votantes. 
Tabla 2

Resultados elecciones '80 a Diputados Provinciales*

$\begin{array}{lll}\text { Partidos PC MAS } & \\ \end{array}$

\begin{tabular}{lccc}
\hline Años & & & \\
\hline 1983 & 0,6 & 0,3 & 0,3 \\
\hline 1985 & - & - & 0,6 \\
\hline 1987 & - & 0,4 & 0,5 \\
\hline 1989 & - & - & 0,8 \\
\hline
\end{tabular}

*Nota: Tabla de creación propia. Resultados tomados de Horacio Lafuente (2007) y Dirección Nacional Electoral.

Continuando con el análisis, en la Tabla 3 el PO se presenta en las elecciones de 1991, y obtiene 594 votos sobre 67.134 votantes. Estas elecciones serán la última en que se presente bajo aquella denominación. Para los siguientes años (1993, 1995 y 1999), conforma un Frente de partidos de izquierdas con el MAS para poder postularse a las elecciones tanto de diputados provinciales (1993) como de gobernador y vicegobernador $(1995,1999)$.

Cabe señalar que la legislación (vigente entre 1991 y 2009) planteaba la caducidad de la personería política de aquellos partidos que no alcanzaran en dos elecciones nacionales sucesivas el $2 \%$ del padrón electoral de su distrito.

Es así que en 1995, el Frente de Unidad Trabajadora (desde ahora FUT) se presenta a elecciones obteniendo 531 votos de los 75.140 votantes en 1995, y en 1999 un total de 764 votos de 89.850 votantes.

\section{Tabla 3}

Resultados elecciones '90 de Gobernador y Vicegobernador**

\begin{tabular}{lcc}
\hline Partidos & PO & $\begin{array}{c}\text { FUT } \\
\text { (Frente de Unidad Trabajadora) }\end{array}$ \\
\hline Años & & - \\
\hline 1991 & 0,9 & 0,7 \\
\hline 1995 & - & 0,8
\end{tabular}

*Nota: Tabla de creación propia. Fuentes de resultados: Ministerio del Interior, Secretaria Electoral, Dirección Nacional Electoral.

En la Tabla 4, mientras el PO recibía 969 votos de los 67.134 votantes, el PC se presentará nuevamente y por única vez en toda la década, recibiendo un total de 411 votos. Como se explicó anteriormente, el FUT tiene su aparición en las elecciones de 1993, obteniendo un total de 1.683 votos de 68.626 votantes, en 19951.232 votos de 75.140 votantes, en 1997 2.609 votos de 82.262 votantes y en 19991.234 votos de los 89.850 votantes.

Es de destacar que en el año 1994 se llevó a cabo en la provincia la reforma de la Constitución. En esa oportunidad el FUT se presentó a la elección de constituyentes y obtuvo dos representantes. 


\section{Tabla 4}

Resultados elecciones '90 a Diputados Provinciales*

\begin{tabular}{lccc}
\hline Partidos & PC & PO & FUT \\
\hline Años & & & \\
\hline 1991 & 0,7 & 1,8 & - \\
1993 & - & - & 2,5 \\
1995 & - & - & 1,9 \\
1997 & - & - & 3,3 \\
1999 & - & - & 1,6 \\
\hline
\end{tabular}

*Nota: Tabla de creación propia. Fuentes de resultados: Ministerio del Interior, Secretaria Electoral, Dirección Nacional Electoral.

En el 2000, aparece un nuevo partido en la provincia: El Movimiento Socialista de los Trabajadores (a partir de ahora MST) que se presentará en las dos categorías analizadas en el trabajo. En las elecciones de 2003 el FUT recibió1.091 votos de 99.345 votantes y en 2007 1.022 votos de 121.381 votantes, el MST en ese mismo año obtuvo un total de 615 votos en su primera presentación.

\section{Tabla 5}

Resultados elecciones '00 de Gobernador y Vicegobernador**

\begin{tabular}{lcc}
\hline Partidos & FUT & $\begin{array}{c}\text { MST } \\
\text { (Movimiento } \\
\text { Socialista de los } \\
\text { Trabajadores) }\end{array}$ \\
\hline Años & 1,2 & - \\
\hline 2003 & 0,9 & 0,5 \\
\hline 2007 & & \\
\hline
\end{tabular}

*Nota: Tabla de creación propia. Fuentes de resultados: Ministerio del Interior, Secretaria Electoral, Dirección Nacional Electoral.

Según la tabla 6, en 2003 el FUT recibe 2.161 votos de los 99.345 votantes. En las elecciones de 2007, el FUT obtiene 2.099 votos de 122.535 votantes y el MST 1.307 votos.

\section{Tabla 6}

Resultados elecciones '00 a Diputados Provinciales*

\begin{tabular}{lcc}
\hline Partidos & FUT & MST \\
\hline Años & & \\
\hline 2003 & 3,0 & - \\
2007 & 2,3 & 1,4 \\
\hline
\end{tabular}

*Nota: Tabla de creación propia. Fuentes de resultados: Ministerio del Interior, Secretaria Electoral, Dirección Nacional Electoral. 
Finalizando con el análisis de los resultados electorales, para las elecciones de 2011 se conforma un Frente de Izquierda de los Trabajadores (FIT) a nivel nacional, fórmula que también se presenta a nivel provincial. En las elecciones de 2011, esta nueva fórmula, obtiene 1.585 votos de 152.725 votantes, mientras el MST recibió 2.605 votos.

Para las elecciones de 2015, el Frente de Izquierda conforma en la provincia una nueva alianza entre el Partido Obrero con la Izquierda Socialista. Reaparece en el marco político el nuevo MAS, obteniendo 205 votos de los 184.364 votantes, el FIT 3.735 y el MST 447 votos.

\section{Tabla 7}

Resultados elecciones '10 de Gobernador y Vicegobernador*

\begin{tabular}{lccc}
\hline Partidos & $\begin{array}{c}\text { FIT } \\
\text { (Frente de Izquierda } \\
\text { de los Trabajadores) }\end{array}$ & MST & MAS \\
\hline Años & & & - \\
\hline 2011 & 1,1 & 1,8 & 0,1 \\
\hline 2015 & 2,1 & 0,2 & \\
\hline
\end{tabular}

*Nota: Tabla de creación propia. Fuentes de resultados: Ministerio del Interior, Secretaria Electoral, Dirección Nacional Electoral.

En la tabla 8, en las elecciones de 2011 el FIT recibe 2.226 votos de los 152.725 . Y en 2015, el MAS obtiene 239 votos, el MST 651 votos y el FIT 6.299 votos de los 184.364 votantes, resultando ser esta última cifra la más alta en cuanto a la categoría de Diputados Provinciales.

\section{Tabla 8}

Resultados electorales '10 a Diputados Provinciales*

\begin{tabular}{lccc}
\hline Partidos & FIT & MST & MAS \\
\hline Años & & & \\
\hline 2011 & 1,9 & - & - \\
2015 & 4,3 & 0,4 & 0,1 \\
\hline *Nota: Tabla de creación propia. Fuentes de resultados: Ministerio del Interior, Secretaria \\
Electoral, Dirección Nacional Electoral.
\end{tabular}

La tendencia al crecimiento se mantiene en el transcurso de los años, a partir del 2000, tanto en la categoría de gobernador y vice como en la de diputados. Siendo más elevado en la segunda de ellas en el orden del 3\%, alcanzando su nivel más alto en 2015 con un 4,8\% si tomamos los partidos de izquierda en su conjunto. Esto nos permite inferir que la presencia de los partidos de izquierda en diferentes espacios de lucha y de debate en la sociedad se ha reflejado en los resultados electorales con un crecimiento notable de los votos en los últimos años.

Con estos primeros resultados electorales, nos enfocamos en las experiencias de los integrantes activos del partido para delinear la organización interna del mismo. Entre los partidos de izquierda que se han presentado como opciones electorales, analizaremos con mayor detenimiento la trayectoria del PO, dada su temprana organización en la provincia y su permanencia hasta la actualidad.

En la entrevista realizada a Miguel del Pla (Anexo 1), dirigente y militante del Partido Obrero, nos describe un panorama de los inicios del partido en la provincia, mencionando una distribución geográfica de las principales localidades en las que se establecen para luego 
presentar a los candidatos en sus primeras elecciones. Entre ellas se encuentran: Río Gallegos, Puerto Santa Cruz, Caleta Olivia y Río Turbio.

Según se desprende de la entrevista realizada al dirigente, los inicios de la organización del Partido Obrero en Santa Cruz se remontan a comienzos del año 1983, en que como parte de una política del partido a nivel nacional, se trasladan a esta provincia un grupo de militantes con la intención de fundar el partido en la región. Luego de la campaña de afiliación se logró legalizar el partido y participar en las elecciones de 1983. En esas primeras elecciones intervinieron también el MAS para la categoría de gobernador y vice, y el PC en diputados.

Tras las primeras dos elecciones en la década de los ochenta, que corresponde a una etapa de organización, observamos que los resultados electorales de la izquierda presentan un incremento durante la década de los noventa, siendo más notable el mismo en la categoría de Diputados Provinciales. Es en este marco de sucesivas presentaciones como minoría política que, en los años 90, participan en la Reforma Constitucional.

Para ampliar un poco más en el tema y su importancia en la esfera política provincial, es en el trabajo realizado por Vilaboa Juan y Hudson Mirna que ambos autores se proponen trabajar en las dos reformas constitucionales ocurridas tanto en 1994 y 1998. En un contexto provincial en el que el PJ-FPV (Partido Justicialista-Frente para la Victoria) y la UCR (Unión Cívica Radical) constituyen una unión para la búsqueda de establecer una permanencia en el ámbito político, el FUT obtiene una activa participación y se convierte como receptores del voto opositor ante esta alianza. En la constituyente provincial de 1994, lograron obtener la elección de dos convencionales: Miguel del Pla y Ricardo Rubé Mercado.

En esta Asamblea, se destacan la presentación de proyectos que buscaban incorporar los derechos jubilatorios y la caja de previsión en el texto constitucional, para que no pudiera ser modificado mediante la legislación, asegurar los recursos naturales de la provincia y la creación de una empresa provincial regulada por los trabajadores, establecer límites para el tiempo de reelecciones en los cargos de gobernador y vice, como así también la defensa de la educación pública, cumpliendo los constituyentes del FUT un rol de voces opositoras en el transcurso de la Asamblea. Sin embargo, con el apoyo de los diputados radicales en su alianza, se llegó a un acuerdo en cambiar la ley electoral del sistema Dhont por el sistema de Ley de Lemas para la renovación de las Cámaras y luego en las elecciones del ejecutivo (Vilaboa y Hudson, 2015).

A partir del debate de la Asamblea Constituyente de 1994, y sus posteriores resultados, se instala la búsqueda de una nueva Reforma para 1998. Con los resultados de la consulta popular, dando como afirmativo el avance de una nueva constituyente, es que el FUT estuvo ausente para integrar y participar de la asamblea.

Sin embargo, como Miguel del Pla señala, las tareas de su partido no se limitan a los momentos de elección. La experiencia de la militancia, como así también las distintas líneas de acción política y sindical en las que intervino el partido, es otro de los rasgos que nos permiten explicar el crecimiento de la izquierda. La variedad de temas que han tocado y puesto en discusión son amplios, intentando así llegar a la mayor cantidad de personas relacionadas a la temática. Es así que nos encontramos con diferentes puntos, entre ellos:

- En los '80, en conjunto con un grupo de militantes de Derechos Humanos, el castigo a los crímenes de lesa humanidad hacia los militares participes de la Dictadura.

- En los '90, el fenómeno de las privatizaciones y los desocupados;

- La lucha por la calidad de vida y de salud;

- Mejoramiento de las condiciones laborales de los trabajadores;

- Condiciones salariales;

- Jubilaciones; 
- El rol de la mujer en la sociedad;

- Calidad educativa (relacionada con los grupos universitarios);

- Y recientemente la protección del medio ambiente.

Pero es importante resaltar la conexión que mantienen con los grupos gremiales y otros movimientos sociales. Se impulsaba la relación con trabajadores de distintos sectores, colaborando entre 1984 y 1988 con la organización del sindicato de panaderos y en la creación de un centro gremial en la UOCRA.

Posteriormente durante los años 90, frente a los cambios producidos como consecuencia de las políticas neoliberales, en el orden provincial plantearon fuertes críticas al sistema político Kirchnerista. Durante esta década, la privatización de empresas como YPF, YCF y Gas del Estado, tuvo importante repercusiones en Santa Cruz debido a la fuerte presencia del estado en la provincia. Según los trabajos realizados por Luque Elida y Martínez Susana, las consecuencias de estas políticas de privatizaciones comienzan a sentirse con más profundidad a partir del año 1994, incrementándose en la década siguiente. En este período es cuando surgen fuertes protestas de grupos de trabajadores desocupados en la zona norte de la provincia, orientada a la actividad petrolera. En estos conflictos y de acuerdo al trabajo de las autoras mencionadas, tuvieron un fuerte protagonismo algunos dirigentes claramente identificados con el FUT y recibiendo apoyo de este partido.

En los últimos años, otro sector ha mantenido una fuerte relación con el PO y una constante presencia. Es el caso de la Asociación de Docentes de Santa Cruz (ADOSAC), como parte de la conducción de la filial local y segunda fuerza a nivel provincial, teniendo también una acción destacada en la localidad de Caleta Olivia con la presencia de dirigentes que han integrado las fórmulas electorales en varias oportunidades.

La estrategia de mayor apertura del partido, que se dio en los últimos años, fue a partir de la constitución de frentes electorales junto a otras fuerzas de izquierda, incluyendo también la alianza sindical con organizaciones que no pertenecen a la misma ideología. El ejemplo más cercano fue la participación en las elecciones de la CTA en el año 2014, que derivó en la formación de una lista para la conducción provincial con gremios estatales (judiciales, docentes y de la salud) y el MST. En lo que respecta a la difusión de las ideas, además de la forma tradicional de distribución de la prensa obrera nacional, se ha incorporado a partir de 2007 (año en que se dio una huelga docente muy importante en la provincia que involucró a otros sindicatos) un programa radial que se difunde una vez por semana con las actividades partidarias y las principales problemáticas locales y regionales.

Finalmente, otro rasgo de apertura y ampliación, se advierte en el crecimiento de la participación de la juventud identificada con ideologías de izquierda dentro de la Universidad, en espacios como los centros de estudiantes.

\section{DISCUSIÓN}

Ante los resultados expuestos, la dinámica interna de las organizaciones políticas y las relaciones con ella, plantea nuevas preguntas. Tal como se había expuesto en un comienzo, las líneas de investigación no toman a los partidos de izquierda como centros de la temática, y los modelos utilizados para estudiarlos se basan en una generalización de los partidos y no específicamente en la conformación de un partido de izquierda. Por lo que se discute si los modelos utilizados para el presente trabajo, aún pueden ser tomados para estudiarlos o si sus aspectos más relevantes pueden ser una guía para la explicación de sus características. 
Pensando en las tendencias de la organización del partido político (sus miembros y dimensiones interiores), habría que desarrollar de qué forma se complementan y si aparecen nuevas formas de organización. Volviendo a los datos electorales, la gran diferencia de votos emitidos hacia los partidos de izquierda, cambian según la categoría: para gobernador/vicegobernador la cantidad es menor que para los diputados provinciales. La diferencia puede residir en una dimensión de ideas, en que la preferencia de aquellos electores se enfoca en la introducción de las temáticas presentadas por los partidos y la defensa de los mismos. Entonces ¿es una búsqueda por parte de los participantes, como nos planteaba Duverger, para introducir la ideología a la legislación? Es una posibilidad que podría tomarse.

Por otra parte, la "necesidad de agruparse" que se hizo mención, desaparece llegando a cuestionar cuán sólida es la estructura interna entre los miembros de un partido y su manera de captar electores por medio de su discurso. Aunque los diferentes partidos de izquierda se agrupen bajo un único concepto de, por ejemplo, Frente, cada partido llega a diferenciarse en sus ideas. Pero eso no significa que, llegado el momento, sus opiniones puedan coincidir en una única motivación para inclinarse a una unión y lucha en conjunto, constituyendo una defensa de los derechos o problemáticas que presenta la sociedad (siendo de carácter económico, político, etc.).

Finalmente, una recomendación para estos términos es complementarlo con futuros trabajos que puedan continuar con esta línea de investigación, centrándonos más allá de los resultados electorales y relacionarlo con la organización de estos partidos.

\section{CONCLUSION}

Por muchos años, la conformación de los partidos de izquierda en la provincia de Santa Cruz fue un proceso de trabajo en diferentes espacios desde dentro del partido (elecciones de dirigentes, adhesión de miembros) como en sus diferentes intervenciones hacia afuera (gremios, agrupaciones de Derechos Humanos, de desocupados, etc.). Con cada una de ellas, llegaron a conformar grupos de debates activos diferenciándose con las formas de acción de los demás partidos de la provincia, planteando cuán importante es la continua organización y la movilización para mantener vigente la presencia del partido y el ideario de izquierda.

Aunque se los presente como minorías en sentido electoral, su metodología de trabajo al incorporarse a los movimientos sociales, defendiendo en diferentes espacios los derechos de los trabajadores, a los sindicatos y aportando un análisis crítico del contexto tanto provincial como nacional, hace de los partidos de izquierda un medio de representación no solo ideológica (con una tendencia trotskista para el caso del PO) sino también de los intereses de aquellos sectores más vulnerables de la sociedad.

Así, fueron organizando una dinámica interna, estableciendo vínculos con los distintos espacios laborales (como es la filial ADOSAC del sector docente, los sindicatos mineros, petroleros) y un posicionamiento ante los problemas sociales del momento (como fue el caso del movimiento de los desocupados a finales de los noventa). El fenómeno político de la Izquierda y su mantenimiento a lo largo del tiempo por diferentes medios, es una forma singular de reconocer estas minorías.

En conclusión, el desarrollo de estos partidos puede observarse más allá de lo electoral que, en semejanza al contexto en que se producen, de manera lenta pero continua permanecen entre las opciones políticas para el electorado, por medio de sus formas de difusión y participación en diferentes espacios de la comunidad. 


\section{REFERENCIAS}

Archivo Histórico Provincial de Santa Cruz.

COGGIOLA O. (2006). Historia del Trotskismo en Argentina y América Latina. Buenos Aires. Ediciones Ryr.

Diarios la Opinión Austral y Correo del Sur

DUVERGER M. (1952). Los partidos políticos. México. Editorial Fondo de Cultura.

LAFUENTE H. (2007). Historia Política de Santa Cruz (1912-1985). Buenos Aires. Editorial Kaikén.

LUQUE E. y MARTÍNEZ S. (2011). Los trabajadores de la zona norte de Santa Cruz frente a la emergencia del movimiento de los desocupados. Trabajo publicado en Sindicatos y desocupados en Argentina 1930/1935 - 1994/2004. Buenos Aires. Dialektik Editora. PIMSA.

SARTORI G. (1980). Partidos y sistemas de partidos. Madrid. Editorial Alianza.

Secretaría Electoral en Dirección Nacional Electoral en el Ministerio del Interior de la República Argentina.

VILABOA J. y HUDSON M. (2015). Las Reformas constitucionales en Santa Cruz en los años noventa. Trabajo presentado en las "II Jornada de Ciencia política y Relaciones Internacionales: Construyendo la política de la teoría a la práctica". Universidad de San Pablo, Tucumán, Argentina. 


\begin{abstract}
ANEXO 1
Entrevista a Miguel del Pla, representante del Partido Obrero, realizada el día 01 de Octubre de 2016 en la ciudad de Río Gallegos, Santa Cruz:

\section{Entrevistador (E): Una Introducción acerca del partido...}

Miguel (M): Nosotros tenemos como partido, una diferencia con los partidos tradicionales porque a los partidos tradicionales se los mide por cuantos sacan de las elecciones, cuantos cargos obtienen. En cambio nosotros tenemos un método de trabajo que le da una permanencia y una posibilidad de hacer un rastreo, desde el punto de vista del trabajo histórico, sobre los planteamientos políticos que esa organización va haciendo. Y es que tenemos un periódico semanal.

Basta mirar el semanario del Partido Obrero, que ya va por el número 1430, creo que estamos en este momento, ¿se entiende? Así que hay 1430 semanas de vida real, donde ahí hubo: qué hicimos frente a un problema, qué hicimos frente a otro, qué dijimos que pasaba, qué dijimos que no pasaba, cómo entendimos cada medida económica, política, social que se fue tomando en Argentina. Gran cantidad de tapas de esos 1400 incluyen fenómenos del interior, debe haber por lo menos 10 o 12 tapas que han salido del tema de Santa Cruz en esa secuencia de 1400 diarios. La tapa de una prensa siempre la sacamos como afiche para que, como vendemos el diario semanalmente, colocamos la tapa como modo de promoción en los locales y en algunos lugares en donde tenemos actividad.

Entonces ahí tenemos una manera de seguir el registro, de por qué una progresa. ¿Por qué progresa o no un partido? Y un partido progresa o no progresa según si, sus caracterizaciones, su modo de comprender la realidad, sus planteamientos, primero tiene que ver con la realidad, es decir, son acertados o no en cuanto a entender lo que pasa y segundo si son formulados de tal manera para que los amplios o no tan amplios pero importantes sectores de la comunidad, con esa manera de entender la realidad, es posible de ser comprendida por los demás. No basta tener razón, hay que ver como uno dice las cosas para ver sí los demás creen que vos tenés razón, y en política el tema no es tener razón sino que los demás digan que vos tenes razón. Es decir, el problema de fondo siempre es como uno comunica las ideas. Bueno, nosotros trabajamos en eso, nuestra historia es la historia de la lucha cotidiana por construir una organización que no se hace en procesos electorales sino todos los días.
\end{abstract}

\title{
E: Podemos continuar con ¿cuándo llegó a Río Gallegos? Su experiencia en la militancia...
}

M: Yo milito en el Partido que antes solía llamarse de otra forma. Política Obrera es una organización que, después continuada como Partido Obrero, se fundó en 1964. Y desde 1964 comenzó a editar un periódico que se llamaba Política Obrera y que fue prohibido en tiempos de la Dictadura. En la Dictadura pasamos a una situación de clandestinidad, yo había empezado a militar terminando mi secundario en el año 69 , prácticamente contemporánea con el Cordobazo, una de las primeras actividades que recuerdo haber organizado un paro de los estudiantes en mi escuela, al día siguiente del Cordobazo. El Cordobazo fue el 29, nosotros organizamos un paro para el día 30 porque ante la represión que había habido en Córdoba, se conmociono al país y hubo un Paro General el día 30 de mayo de 1969. 
Yo vivía en Hurlingham, en el Gran Buenos Aires. Viviendo allí, en nuestra escuela hacía ya varios meses que habíamos empezado a organizarnos, un grupo de gente que estábamos viendo la necesidad de empezar a participar en política y luchar, y plantearnos reclamos sociales. Y fue el primer paro que hicimos, la primera acción que chocábamos directamente con las autoridades escolares. Recordemos que estábamos en una Dictadura, no en la última sino en la anterior, en ese tiempo aún estaba Onganía.

Así que bueno, empezamos ahí. Cuando llegó la Dictadura, Política Obrera fue prohibida como organización, hubo un decreto de la Dictadura que decía que varios partidos, en particular nos nombraban específicamente a nosotros, que incluso no habíamos tenido en el período del 73 al 76 personería legal, no habíamos llegado a legalizarnos como organización electoral. Teníamos una actividad, éramos conocidos por una minoría de gente, muy chiquito todavía, estábamos en cuatro o cinco ciudades de la Argentina pero ya existíamos, y el poder político nos tenía identificados, incluso en el período de la Triple A varios compañeros asesinados.

Bueno, entonces cuando nos prohibieron nosotros trabajamos en la clandestinidad como Política Obrera, teniendo nuestro diario varios nombres durante la Dictadura para ir eludiendo la persecución, y se imprimía en la clandestinidad, nos rompieron una imprenta y fue un problema, no sólo por los compañeros que fueron presos por diferentes motivos sino también teníamos que buscar la manera de volver a editar, que durante la Dictadura no salía semanalmente sino cada mes o cada quince días, y era repartido de forma disimulada: en un paquete de yerba, bolsas de residuos, doblado el diario.

Pero mantuvimos el nivel de organización, durante la época de la Dictadura creció. Y se desarrolló. Llegamos a localidades, en un trabajo muy clandestino. Ahora, cuando se salió de la Dictadura, entró una crisis profunda de problemas sociales, se habilitó la posibilidad de legalizar el partido, entonces nosotros vimos una oportunidad, ésa alternativa de participar. Cuando se llegó esta posibilidad de organizar el Partido Obrero en otras provincias, yo estaba viviendo en el Gran Buenos Aires y trabajaba como metalúrgico. Como Política Obrera seguía prohibida y para legalizarnos debíamos presentarnos con otro nombre, nuestra prensa histórica pasó a llamarse Prensa Obrera, por un Partido Obrero. La idea era que queríamos formar el Partido Obrero, para las masas trabajadoras.

Ahí salió la oportunidad de irse a otras provincias. En febrero del año 83, subimos a un auto con un grupo de compañeros y venimos, con la idea de legalizar el partido. Llegamos a Puerto Santa Cruz, a la casa de un compañero, comenzando con una campaña de afiliación e iniciamos todos los trámites legales para conseguir los 222 afiliados para que sea legal en Santa Cruz. Lo conseguimos en menos de un mes, había una inquietud política al salir de la Dictadura que nos decíamos "hay que participar". Con el resultado de esta actividad, nos trasladamos a Río Gallegos, y sinceramente no sabía que iba a quedarme a vivir en Santa Cruz.

Finalmente, el progreso fue tan rápido y tan impactante que se logró estructurar un partido y presentarnos en la primera elección en cuatro o cinco localidades. Llegamos a presentar en Caleta Olivia, en Río Turbio, en Río Gallegos y en Puerto Santa Cruz, la cual fue una experiencia bastante interesante. Y me quedé a organizar al partido. 


\section{E: A partir de que se estableció en Río Gallegos ¿de qué manera se organizó con los demás compañeros?}

M: Principalmente participando en los reclamos sociales y populares en varios rubros.

\section{E: ¿Qué temas destacaban?}

M: Derechos Humanos. Nos metimos en las Comisiones por la cual luchaban por el castigo de los militares, tema con poco movimiento en Río Gallegos, pero un grupo de activistas importantes que también se integraron al partido. Otra actividad importante es la creación de un centro gremial en la UOCRA para los trabajadores de la construcción.

En el año 84 al 88 ayudamos con la presencia de un sindicato de panaderos. En los '90 fuimos en contra de las privatizaciones y el combate de la instalación como sistema político del régimen kirchnerista, siendo críticos ante sus decisiones.

En la Convención Constituyente de 1994, pensamos llevar a la discusión problemas de la población, tomando un eje que nos permitió presentar un proyecto que incorporaran los derechos jubilatorios y la caja de previsión en el texto constitucional para no fuera modificado.

Estos fueron los primeros pasos desde el 83 al 94 en la lucha de los derechos en la provincia de Santa Cruz. No bajamos el $2 \%$ desde entonces y no teníamos que estar preocupándonos que nos sacaran la personería.

\section{E: Me interesa saber cómo se relaciona el Partido Obrero con otras fuerzas de izquierda, ¿cómo es la relación actualmente?}

M: Actualmente en Santa Cruz somos Partido Obrero, a diferencia de años anteriores. Es novedad en Argentina que se formó un Frente de Izquierda, el cual su primera presentación fue en 2011, llegando a ser un proceso nacional y no lo que pasa en Santa Cruz.

Pero el Partido Obrero ha ganado cierta relevancia, llegando a ser el más conocido con la introducción de dos concejales de la elección del año pasado, la participación en diferentes cargos gremiales, en el gremio docente como una segunda fuerza, llegando a estar insertados en la vida real.

El Frente de Izquierda es una experiencia muy importante, porque nos juntamos a nivel nacional con el PTS y con Izquierda Socialista, la cual logró tener una personalidad legal en Santa Cruz pero aún es muy reducido su trabajo.

Se empezó a visualizar a la Izquierda como una alternativa política en el consenso nacional. Nosotros somos constructores de esa Frente y estamos convencidos de que para que la Izquierda se desarrolle logre unirse. De momento somos esos tres partidos, también está el MAS pero ciertamente es difícil encontrar un punto de equilibrio. Pienso que a largo plazo nos tenemos que unir todos, es cuestión de tiempo para lograr que nos unamos en una fuerza de Izquierda revolucionaria.

No es que no tenemos vinculación, en la conducción de la CTA lo compartimos con el MST, y otras organizaciones. Son cuestiones que hemos llegado a madurar dejando de lado rasgos de sectarismos y cada uno tiene una opinión distinta o motivos diferentes, y de esta forma no impide otros puntos de unión. 